REVISTA

MEXICANA DE

ECONOMÍA Y

FINANZAS

REMEF

(TIIE MEXICAN JOURNAL OF

ECONOMICS AND FINANCE

\section{Revista Mexicana de Economía y Finanzas, Nueva Época}

Volumen 16 Número 4, Octubre - Diciembre 2021, pp. 1-27, e527

DOI: https://doi.org/10.21919/remef.v16i4.527

(Recibido: 20/julio/2020, aceptado: 3/noviembre/2020, publicado, 18/marzo/2020)

\title{
La productividad del sector manufacturero: caso Colombia 2005-2016
}

\author{
Diana L. Becerra-Peña ${ }^{1}$ - Universidad de Guadalajara, México \\ María Ximena Lemos Mejía² - Universidad EAN, Colombia
}

El objetivo de este trabajo es analizar empíricamente la evolución de la productividad total de los factores (PTF) en la industria manufacturera colombiana de 2005 a 2016, a través de la estimación del Índice de Malmquist (IM), con un panel de veintinueve subsectores productivos. Los resultados muestran una caída promedio en la PTF de la manufactura colombiana de $1.1 \%$, derivado de un retroceso en el componente tecnológico. Se recomienda a los hacedores de política promover el uso de Tecnologías de la Información y la Comunicación, así como innovaciones tecnológicas. La limitación encontrada corresponde a la falta de información desagregada a nivel empresa de la totalidad de unidades económicas del sector durante el período de análisis. La originalidad de la investigación recae en el monitoreo de los cambios en la productividad de la manufactura y su descomposición en eficiencia y tecnología a lo largo del tiempo. En conclusión, durante el período de estudio se vislumbra una desindustrialización temprana del sector manufacturero, donde los componentes de eficiencia y de tecnología no fueron en la misma dirección.

Clasificación JEL: C43, D24, L60.

Palabras clave: productividad total de factores, Índice de Malmquist, sector manufacturero, industria manufacturera, Colombia.

\section{The Productivity of the Manufacturing Sector: The Case of Colombia 2005-2016}

The objective of this work is to empirically analyze the evolution of total factor productivity (TFP) in the Colombian manufacturing industry from 2005 to 2016, through the estimation of the Malmquist Index (MI), with a panel of twenty-nine productive sub-sectors. The results show an average fall in the TFP of Colombian manufacturing of $1.1 \%$, derived from a setback in the technological component. It is recommended for policy makers to promote the use of Information and Communication Technologies, as well as technological innovations. The limitation found corresponds to the lack of disaggregated information at the company level for all economic units in the sector during the analysis period. The originality of the research lies in the monitoring of changes in manufacturing productivity and their decomposition into efficiency and technology over time. In conclusion, during the study period an early de-industrialization of the manufacturing sector can be seen, where the components of efficiency and technology were not in the same direction.

JEL Classification: C43, D24, L60.

Keywords: total factor productivity, Malmquist Index, manufacturing sector, manufacturing industry, Colombia.

1 Autor de correspondencia. Departamento de Métodos Cuantitativos, Centro Universitario de Ciencias Económico Administrativas. Periférico Norte $\mathrm{N}^{\circ}$ 799, Núcleo Universitario Los Belenes, C.P. 45100, Zapopan, Jalisco, México. diana.bp@academicos.udg.mx ORCID: https://orcid.org/0000-0002-2796-3777

2 Facultad de Administración, Finanzas y Ciencias Económicas. mxlemos@universidadean.edu.co

*Sin fuente de financiamiento para el desarrollo de la investigación. 


\section{Introducción}

Después de la década del noventa y hasta la actualidad, la economía colombiana ha presentado un sesgo importante hacia la producción y exportación de materias primas e hidrocarburos, esto en detrimento de su industria manufacturera, la cual ha caído notable y persistente del nivel de sus exportaciones y del valor agregado que genera.

Posterior a la crisis de 2008, ha sido la industria manufacturera la que más ha tenido dificultad para recuperarse y poder retomar, al menos, tasas de crecimiento similares a los años previos a la crisis. Diversos autores (De Gregorio, 2018; Gómez, 2015; Haltiwanger \& Eslava, 2017) han atribuido tal pobre desempeño, principalmente, a la incapacidad de la industria de ser eficiente, así como a su carente enfoque en el mercado internacional.

Si analizamos las cifras que el Departamento Administrativo Nacional de Estadística presenta de las Cuentas Nacionales en 2016 sobre producción real de las diferentes ramas económicas, se observa que, en Colombia, la manufactura supera al resto de actividades económicas en alrededor de 35 por ciento. Sin embargo, la capacidad e intensidad de la industria por generar valor agregado al total de la economía son mucho menores en la manufactura comparado con sectores como los Servicios Financieros, y presenta una consistente tendencia a la baja (DANE, 2017).

Este indicador preocupa si se tiene en cuenta que desde 2003 el Ministerio de Comercio, Industria y Turismo junto al Consejo de Competitividad han llevado a cabo importantes esfuerzos para dinamizar la manufactura colombiana enfocándose en las capacidades tecnológicas para generar valor agregado. A través del ofrecimiento de créditos institucionales para la compra de maquinaria especial y equipo tecnológico y la disminución de costos laborales para el empleador, los proyectos buscan mejorar la eficiencia y la competitividad de las empresas manufactureras, principalmente, en el mercado de exportación (CPC, 2016).

A pesar de esto, desde 1990 la industria manufacturera no ha sido representativa en los indicadores económicos más relevantes del país. Por ejemplo, el empleo generado por el sector ha sido de los más bajos de toda la economía, oscilando entre 12 y 15\%, y no ha logrado atraer una mayor cantidad de mano de obra migrante del campo y la ha desplazado hacia el sector de servicios y de comercialización a lo largo de las décadas. Como sugiere Kaldor (1975) la baja participación del empleo formal en el sector manufacturero además de ser un síntoma de desindustrialización, también evidencia que su persistencia en el tiempo propicia el descuido de la manufactura por trasladar mano de obra hacia el sector primario y terciario, derivando en una desindustrialización prematura (Martínez \& Ocampo, 2011).

Ante un escenario de desindustrialización temprana, las repercusiones que este conlleva para una economía como la colombiana son trascendentales y devastadoras. Dado que la principal fuente de ingresos de Colombia se encuentra en la producción y exportación de commodities, las divisas originadas por dichas exportaciones traen consigo una apreciación real en el tipo de cambio que, básicamente, destruye a la industria local al abaratar las importaciones y encarecer las exportaciones de otras industrias distintas a las de commodities y, naturalmente, disminuyendo la tasa de ocupación en el sector manufacturero (Clavijo et al., 2012). De igual manera, un pronto debacle de la manufactura en el país generaría, además de desempleo, poca o nula independencia económica y le 
haría más vulnerable a las condiciones externas del mercado, marginando su capacidad de acción ante las eventuales crisis, como las ocurridas en 2008 y la actual pandemia global.

Durante el período de 1975 a 2016, las exportaciones colombianas totales han mostrado una tendencia creciente, aunque, lenta e inestable en los últimos años, promovidas por el enfoque de la economía en su sector de servicios y de hidrocarburos. Las exportaciones tradicionales (hidrocarburos y café) han rezagado en los últimos diez años a las exportaciones no tradicionales (manufacturas), dada la fuerza que ejercen los precios internacionales del petróleo y el carbón y que hacen atractivo concentrar, hoy, el 90\% del total de exportaciones tradicionales colombiana, e incluso marcar el comportamiento general de las exportaciones totales.

A nivel de manufactura, para 2016, las exportaciones industriales estaban constituidas principalmente por la industria de productos alimenticios (27\%), refinación de petróleo y coquización (productos básicos) (14\%), productos y sustancias químicas (15\%) y productos metalúrgicos (14\%), donde las empresas que conforman a las tres primeras industrias son en un 85\% empresas de capital extranjero. También es de señalar que, las industrias de Productos alimenticios y la de Productos y Sustancias químicas son industrias que recientemente se han adherido (en 2013) al programa de transformación productiva llevado a cabo en el marco de la Política Industrial colombiana.

De acuerdo con el Consejo de Competitividad (CPC, 2016) esta adhesión sectorial se dio al evidenciarse una participación importante de estos sectores en las exportaciones no tradicionales totales y a su crecimiento acelerado. Ello muestra que la decisión de apoyo sectorial no correspondió a un genuino interés por crear tejido productivo en la economía (ya que industrias vitales como el sector químico conllevaría al desarrollo de los sectores de caucho, plástico o farmacéuticos), sino a una ventaja observado y que lograron estos sectores individualmente, sin el amparo de los entes encargados de su desarrollo.

Aún, al interior de Colombia aún no existe un tejido productivo ni sólido ni, mucho menos, consistente, pues las políticas hasta hoy no han profundizado en el entendimiento de la industria, y se han basado casi que exclusivamente en la coyuntura y sus indicadores. Por todo lo anterior, se vuelve clave abordar el problema de la industria manufacturera desde la productividad y resolver las siguientes preguntas de investigación: ¿qué tan productivos han sido los subsectores de la manufactura colombiana?, ¿cómo ha evolucionado la productividad de los subsectores?, ¿cuál es la fuente de los cambios en la productividad de los subsectores? Por ello, el objetivo en este trabajo es analizar empíricamente los cambios en la productividad total de los factores (CPTF) en el sector manufacturero en Colombia.

Para responder a las preguntas antes planteadas, se estima el índice de Malmquist (IM), que asume rendimientos constantes a escala, en un panel de veintinueve subsectores manufactureros con información de 2005 a 2016. Entre las ventajas de este índice se encuentra la estimación de los cambios en la productividad entre dos períodos de tiempo, por tanto, para dar seguimiento a esos cambios es imperante el uso de un panel de datos con información de al menos tres años.

Otra de las bondades que posee el IM es la descomposición de los CPTF en un componente de eficiencia y un componente de tecnología, con ello se responde al tercer interrogante acerca de la fuente de los CPTF. Se esperaría que la productividad de los subsectores manifieste cambios positivos y que el origen de estos radicara en el componente de eficiencia, de lo contrario se confirmaría una desindustrialización temprana de la manufactura colombiana. 
En este sentido, conocer cuál es la fuente de los cambios en la productividad en el tiempo abona a mejorar la formulación de mejores políticas. Por ejemplo, si se observa un bajo crecimiento de la productividad derivado de un avance del componente tecnológico, la mejor recomendación sería fomentar innovaciones tecnológicas que ayuden a desplazar la frontera de producción hacia arriba (Kim \& Han, 2001). De ahí la relevancia y la originalidad de esta investigación.

La estructura del documento es como sigue, la sección dos incluye un breve bosquejo sobre la literatura que analiza la productividad, con énfasis en el sector manufacturero, tanto en el contexto internacional como en el nacional. En la sección tres se desarrolla la propuesta metodológica de la investigación, se muestra el origen de los datos y las variables utilizadas. Los resultados del análisis y su discusión se presentan en la sección cuatro. Finalmente, se cierra con un apartado de conclusiones.

\section{Estado del Arte}

\subsection{Enfoques para estimar la eficiencia y la productividad}

Desde inicios del siglo XIX se comenzó a relacionar los insumos con los productos, posteriormente surge diversa literatura sobre la estimación de las funciones de producción, donde gran parte de ella reside en la comprobación de implicaciones con la tecnología y los comportamientos optimizadores. De lo anterior se desprende el interés por conocer las relaciones que guardan los niveles de insumos con los niveles alcanzados de productividad.

Existen gran diversidad de enfoques metodológicos para estimar la eficiencia en un sentido amplio, donde destacan los métodos de frontera que pueden clasificarse como: no paramétricos, paramétricos y semi-paramétricos ${ }^{3}$.

La optimización matemática es la base para la construcción de la función de producción en los métodos no paramétricos, su popularidad recae en que no precisan de una forma funcional previa. El Análisis Envolvente de Datos (DEA, por sus siglas en inglés: Data Envelopment Analysis) destaca dentro de este grupo, ha tenido numerosas aplicaciones dentro de los sectores público y privado derivado de su adaptación a contextos con múltiples insumos y múltiples productos, se basa en la programación matemática, además no requiere supuestos sobre la distribución de la ineficiencia.

Por el contrario, las técnicas paramétricas precisan la especificación de la función de producción a través de parámetros constantes ayudados de métodos econométricos, como puede ser la función Cobb-Douglas o la función translogarítmica. Estas técnicas pueden ser determinísticas, si las desviaciones de la frontera se explican por la ineficiencia, o estocásticas, si las desviaciones de la frontera se descomponen en ineficiencia y ruido, como en el caso del Enfoque de Frontera Estocástica (SFA, por sus siglas en inglés: Stochastic Frontier Approach), una de las técnicas más frecuentes dentro de los paramétricos.

\footnotetext{
${ }^{3}$ Para revisar con mayor profundidad las distintas técnicas utilizadas en la estimación de eficiencia ver los trabajos de Álvarez (2001), Murillo-Zamorano (2004), Coelli et al. (2005), Andor y Hesse (2014) y Aparicio, Lovell y Pastor (2016).
} 
Mientras que los métodos semi-paramétricos son una mezcla de las técnicas paramétricas y no paramétricas. Tal es el caso del método envolvente estocástico no lineal de datos (StoNED, por sus siglas en inglés: Stochastic Non-smooth Envelopment of Data), que combina propiedades de DEA y SFA, puesto que no precisa ninguna forma de la función de producción y las desviaciones de la frontera se descomponen en ineficiencia y ruido, respectivamente (Schaefer \& Clermont, 2018).

Bajo este escenario, se destaca que la incorporación de números índice se da a un ritmo menos acelerado si se compara con las técnicas anteriores. Aquí sobresale el IM, a partir de técnicas no paramétricas, que permite medir la productividad con la ventaja de ser capaz de descomponerse en medidas de cambio de eficiencia y de cambio tecnológico, respectivamente, como se explica en la Sección 3.

\subsection{Análisis del desempeño en el sector manufacturero}

En la revisión de literatura se encontraron aplicaciones previas que analizaron el sector manufacturero dentro del contexto internacional a nivel país que se resumen a continuación.

Bajo el enfoque paramétrico, con la técnica SFA, Kim y Han (2001) estudian 508 empresas en Corea, que cotizan en la Bolsa de Valores durante 1980-1994 y clasificadas en 7 industrias (alimentos, textiles, papel, química, no metálica, metales básicos y fabricación). Los autores encontraron que el progreso tecnológico es la fuente del crecimiento de productividad.

Pavnick (2002) analiza el caso de las plantas manufactureras chilenas durante 1979-1986 para analizar la evolución de la productividad, con una función Cobb-Douglas, y la relación que guarda con la liberalización del comercio. Sus resultados muestran que la manufactura chilena creció luego de la liberalización del comercio, debido a la reorganización de los recursos al interior de la economía.

Posteriormente, Fernandes y Paunov (2012) utilizan información 4,913 firmas chilenas del sector manufacturero de 1992 a 2004 para analizar la relación que guardan la productividad y la inversión extranjera directa (IED) en servicios. Sus resultados indican que la IED da oportunidad de que las empresas rezagadas compitan con los líderes de la industria, ello implica que la IED estimula actividades innovadoras en las empresas manufactureras.

En Turquía, Önder, Deliktaş y Lenger (2003) utilizan datos para dieciocho provincias entre 1990 y 1998. Sus resultados indican que la manufactura del sector privado es más eficiente que el sector público. En Brasil, Muendler (2004) con información de 9500 empresas medianas y grandes durante 1986-1998, encontró que la competencia extranjera incrementa la productividad y que el uso de insumos extranjeros tiene un impacto menor en la productividad.

El caso de Vietnam es estudiado por Ha, Kiyota y Yamanouchi (2016), con datos a nivel empresa, que incluyen a las de propiedad del Estado y extranjera sin excepción de su tamaño, para estimar la PTF de 2000 a 2009, con una función de producción tipo Cobb-Douglas, y la comparan con los resultados de China, India, Japón, Tailandia y Estados Unidos. Los autores concluyen que Vietnam tiene una ineficiente asignación de recursos, como ocurre en una economía en desarrollo, asimismo señalan que los posibles incrementos de la productividad originados por la eliminación de distorsiones en la industria son grandes.

Respecto al comportamiento de las economías en desarrollo como Vietnam y su estrategia de crecimiento basado en el sector manufacturero, la evidencia que presenta de Souza \& GómezRamírez (2018) es reveladora sobre todo en estos tiempos en el que el comercio mundial se basa en 
las cadenas globales de valor (CGV's) y la deslocalización internacional de la producción. Los autores utilizando matrices input-output y apelando a la paradoja del crecimiento del caso mexicano, evidencian que las economías en desarrollo ven distorisionados sus sectores manufactureros por una liberalización rápida del comercio que sustituye al consumo de bienes intermedios nacionales y los sustituye por los extranjeros para la fabricación de los bienes de exportación. Esta sustitución impacta negativamente la demanda interna de bienes intermedios nacionales y, por lo tanto, relentiza el crecimiento generalizado del sector manufacturero del que hacen parte puesto que, prima la compra desde el exterior y elimina la competencia local, desencadenando una desindustrialización premuarura (de Souza \& Gómez-Ramírez, 2018; Rada, 2007) trayendo problemas serios de desempleo e informalidad que requieren de una efectiva y eficiente intervención estatal para su relocalización.

Esta cadena de efectos cuestiona la estrategia de crecimiento de las economías duales en vías de desarrollo máxime, cuando estas adolecen de industrias de avanzada y se centran en satisfacer sectores, principalmente, maquiladores que ocupen la mano de obra disponible generalmente de informal (Basole \& Narayan, 2020). A partir del seguimiento que hacen Basole y Nayaran (2020) a ciertos indicadores de crecimiento y empleo en India durante 1983-2017 y usando la Prueba BaiPerron, los autores detectan que el sector manufacturero indio es un gran receptor de mano de obra sobrante en el sector primario, contratándola, principalmente, de manera informal para aumentar su producción. Sin embargo, los autores también muestran que una gran parte de la mano de obra disponible migra hacia actividades de servicios, poniendo en duda la urgente necesidad de un sector manufacturero como agente de crecimiento económico.

Ante este paradigma dual del empleo formal e informal, la productividad de la industria manufactuera dependerá, pues, de la forma en que se incorpore capital tecnológico y bienes intermedios para que interactúen con la mano de obra disponible (Basole \& Narayan, 2020) y hagan ganar eficiencia, siendo en este sentido determinante la calidad de la mano de obra. De igual forma, existe evidencia que indica que la productividad manufacturera empeora cuando la mano de obra utilizada es informal, ya que usualmente esta se caracteriza por su baja calidad y su utilidad de encuentra en industrias de baja complejidad y serivicos que requieren mano de obra barata como el ensamblaje (F. Álvarez et al., 2018; OIT, 2017; Puyana \& Romero, 2013).

Bajo el enfoque no paramétrico, se encuentra el trabajo de Mahadevan (2002) sobre veintiocho subsectores de la manufactura en Malasia desde 1981 a 1996. Los resultados del DEA identifican un bajo crecimiento de la PTF derivado de pequeños incrementos tanto de eficiencia como de tecnología.

Por su parte, el estudio de Kumar (2006) se acota a la industria manufacturera de quince de los principales estados de la India en el período entre 1982-1983 y 2000-2001. Las estimaciones en la India indican que, en general, hubo una mejora en la PTF regida por el progreso tecnológico, donde este último presenta un sesgo de utilización de capital, además de las diferencias regionales en la PTF.

Otro punto de interés es la eficiencia energética dentro del sector manufacturero. Uno de los trabajos a destacar aquí es el de Zhang, Lundgren y Zhou (2016), donde analizan 3066 empresas de 14 subsectores (pulpa y papel, hierro y acero, productos químicos, piedra y minerales, minería, 
alimentos, productos de madera, vehículos de motos, maquinaria, caucho y plástico, electrodomésticos, textiles, productos metálicos e impresión) durante 2001-2008 con DEA. Sus resultados indican la posibilidad de mejorar la eficiencia energética en todos los subsectores.

En el uso de la metodología del IM, destaca el trabajo de Leung (1998) que explica la PTF de la manufactura en Singapur con datos de treinta industrias durante 1983 a 1993. Su estimación muestra un crecimiento anual de $4.6 \%$, además comprueba que la propiedad extranjera y la calidad de la mano de obra tienen un impacto importante en los CPTF.

Bajo el mismo enfoque, Lee, Kim y Heo (1998) emplean datos de treinta y seis sectores manufactureros en Corea entre 1967 y 1993. Se encontró que la PTF mejoró 0.286\% en conjunto a lo largo del período de estudio, originado por un avance en el componente tecnológico (1.141\% anual) con pérdida de eficiencia ( $0.855 \%$ anual), ello implica un beneficio de la innovación tecnológica de la economía coreana, donde los sectores con rezago en tecnología manifestaron cierto atraso.

Karadağ, Önder y Deliktaş (2005) estiman la PTF de la manufactura en un conjunto de provincias Turcas durante 1990-1998. Sus resultados indican que gran número de provincias tuvieron mejoras en la PTF del sector público, pero sólo la mitad de las provincias tienen desempeño favorable en el caso del sector privado.

Destaca el trabajo de Kalai y Helali (2016) por emplear un panel de seis sectores manufactureros en Túnez a lo largo de cincuenta años (1961-2010). Sus estimaciones indican que la fuente principal de la falta de productividad se encuentra en el componente tecnológico, a pesar de que el cambio en los niveles de eficiencia fue satisfactorio.

Por su parte, Lin y He (2018) estudian la industria manufacturera tradicional (dieciocho subsectores) en Beijing durante 2000-2014. Sus estimaciones concluyen que el crecimiento de la productividad se debe, en gran medida, al progreso tecnológico, a pesar de registrarse una pérdida de eficiencia, únicamente tres de los subsectores consiguieron mejoras en la PTF.

Mientras que, Shi, Wang y Zhu (2019) analizan los cambios en la productividad de 671 empresas manufactureras chinas que cotizaron en la Bolsa de 2009 a 2014. Sus resultados indican que la investigación y desarrollo (I+D) podrían mejorar la eficiencia de los cambios tecnológicos.

Por su parte, Boyd y Lee (2019) estiman la eficiencia de las energías eléctrica y térmica en cinco industrias manufactureras basadas en el uso de metales (fabricación de productos de metal, maquinaria, computadoras y productos electrónicos, equipo de transporte, equipo eléctrico) en Estados Unidos durante 1987-2012, a través del SFA y del IM. Se encontró que la eficiencia eléctrica es mejor que la eficiencia térmica.

Finalmente, Day y Farid (2019) acotan su trabajo al estudio del desempeño de los diez principales fabricantes de automóviles en todo el mundo durante 2011-2016 con ayuda del IM. Sus resultados indican que hubo un incremento en la productividad de la industria durante el período de estudio, donde destacaron, en orden de ganancias, Toyota, Volkswagen y Mercedes-Benz.

En el caso de la comparación entre países, destaca el trabajo de Eberhardt y Teal (2010) que, con una función de producción tipo Cobb Douglas, estudian 48 países desarrollados y en vías de desarrollo entre 1970 y 2002. Los autores señalan que las diferencias de tecnología son relevantes para la comprensión de la diferencia entre países con respecto de la productividad.

El interés por estudiar el comportamiento del sector manufacturero en Colombia se destaca dentro de la revisión de literatura, como se muestra a continuación. Pombo (1999) utiliza el residuo de Solow para analizar noventa y cuatro sectores manufactureros de 1970 a 1995, sus estimaciones 
señalan la desaceleración del crecimiento de la productividad a mediados de los setenta hasta tornarse negativo en 1980, con una recuperación cinco años después y a partir de ello ha presentado ganancias moderadas.

Bajo la metodología de Mínimos Cuadrados Ordinarios (MCO), Ramírez (1995) analiza 2,574 firmas que permanecieron en el sector manufacturero entre 1974 y 1987. Sus resultados muestran que las empresas con mayor eficiencia son las más antiguas, con mayor mano de obra calificada y que se ubican en sectores con mayor propensión a exportar y mayor tasa de protección efectiva. Por su parte, Meléndez et al. (2003) estudian 21 industrias del sector manufacturero entre 1977 y 1999 , destacan que las reformas comerciales sugieren un crecimiento de la productividad, especialmente en el sector textil, papel, metales básicos y maquinaria, mientras que el sector del vidrio fue el único con crecimiento sostenido en la productividad durante todo el período de estudio.

A través del modelo de frontera estocástica, Acevedo y Ramírez (2005) identifican disparidades regionales del subsector de fabricación de confecciones durante 1992-2001, en once departamentos seleccionados. Los resultados muestran que las empresas producen por debajo de su frontera, con una tendencia creciente del nivel agregado de eficiencia, siendo la aglomeración regional un factor determinante de esta. Con este mismo enfoque, Barrientos, Tobón y Gutiérrez (2009) analizan la eficiencia del subsector de calzado y marroquinería de cuero en Bogotá, Bucaramanga, Cúcuta y Medellín durante 2007. Sus resultados indican que la eficiencia de las empresas está relacionada con las escalas de los rendimientos del factor trabajo.

Por su parte, Echavarría, Arbálaez y Rosales (2006) en un panel desbalanceado de plantas durante 1981-1992 a través de una función de producción logarítmica. Los autores muestran que la productividad multifactorial no cae entre 1980 y 1990, aunque el crecimiento fue mayor durante los noventa debido al impacto de las reformas económicas, así como por la apertura hacia adentro.

Respecto al papel que juega el empleo en la productividad manufacturera, Rodrik (2013) utiliza modelos de convergencia para argumentar que las economías con una mayor cantidad de empleo en el sector manufacturero crecen más rápido debido a un catch up que sucede de la productividad laboral en los sectores industriales que moviliza a estos últimos hacia una frontera eficiente de productividad y les hace, incluso, menos dependientes de variables no controlables como las políticas internas, las calidad institucional o la geografía.

Sin embargo, para Felipe et.al (2019) existe una percepción creciente de que, en los últimos tiempos se ha hecho más difícil para las economías mantener altos niveles de producción y empleo manufacturero mientras aumentan simultáneamente los salarios y el nivel de vida, lo que haría que el empleo alcance un punto en declive y por tanto, la participación de la manufactura de la economía en general. No obstante, los autores también señalan que en la medida en que las economías experimentan aumentos en los salarios pueden sustituir el trabajo por capital y este cambio debería ser más evidente por la creación de empleo manufacturero que por la participación de la manufactura en la producción (Felipe et al., 2019), situación que poco o nada, sucede para el caso colombiano.

Finalmente, Delgado (2019) estima la eficiencia técnica de 1,612 empresas manufactureras colombianas durante 2013-2015, con una función de producción Cobb Douglas. Concluye que 83.6\% de las empresas mantuvieron niveles de eficiencia debajo de la mitad del potencial tecnológico del sector, siendo la producción de bebidas y minerales no metálicos los promedios más altos, se 
concluye que la productividad de estas empresas depende del uso eficiente de su capital y su recurso humano.

\section{Metodología}

\section{1 Índice de Malmquist}

La estimación de los cambios en la productividad total de los factores en la industria colombiana se realiza a través de la metodología del índice de Malmquist, desarrollado inicialmente por Malmquist (1953) como resultado de una proporción de dos funciones de insumos, para expresar una cantidad de consumo o nivel de vida dentro del contexto del consumidor. La primera aplicación del IM fue realizada por Moorsteen (1961) a través de una comparación del insumo de una misma empresa en dos momentos diferentes del tiempo.

Posteriormente, Caves, Christensen y Diewert (1982) aplicaron la metodología del IM para estudiar el caso de dos empresas durante un mismo período de tiempo, a partir de su trabajo se consolida la metodología del IM (también conocido como el Índice de Productividad de Malmquist) con el cual argumentaron que existen dos formas de estimar las diferencias en la productividad: con un enfoque orientado al insumo y con un enfoque orientado al producto.

En esta investigación utilizaremos un IM orientado al producto, donde la productividad se define como la razón estimada entre la cantidad de productos y la cantidad de insumos empleados. Al tratarse de un número índice se desprende de los métodos no paramétricos, con lo que se permite la descomposición de los CPTF en un término de eficiencia y en un término de tecnología como se explica a continuación.

Una unidad tomadora de decisiones (DMU, por sus siglas en inglés Decision Making Unit) que produce un conjunto de productos $y=\left(y_{1}, \ldots, y_{M}\right) \in \mathbb{R}_{+}^{M}$ a partir de un conjunto de insumos $x=$ $\left(x_{1}, \ldots, x_{N}\right) \in \mathbb{R}_{+}^{N}$. Donde el conjunto de tecnología $S^{t}$, definido como el conjunto de todas las combinaciones factibles de insumos y productos, que caracteriza la tecnología de la DMU disponible en el periodo $t$ de la siguiente manera:

$$
S^{t}=\left\{(x, y) \in \mathbb{R}_{+}^{M+N}: x \text { puede producir } y\right\}
$$

Se asume que la tecnología cumple con las siguientes condiciones: (C1) $S^{t}$ es cerrado y no vacío. (C2) si $y \geq 0_{M}$, entonces existe una $x$ tal que $(x, y) \in S^{t}$. (C3) si $(x, y) \in S^{t}$ y $x^{*} \geq x$, entonces $\left(x^{*}, y\right) \in S^{t}$. (C4) si $y>0_{M}$, entonces $\left(y, 0_{N}\right) \notin S^{t}$. (C5) si $x \geq 0_{N}$ y $(x, y) \in S^{t}$, entonces existe un límite finito $b \geq 0$ tal que $0_{M} \leq y \leq b \times 1_{M}$. (C6) si $x>>0_{N}$, entonces existe $y>>0_{M}$ tal que $(x, y) \in$ $S^{t}$. Y (C7) si $(x, y) \in S^{t}$ y $0_{M} \leq y^{*} \leq y$, entonces $\left(x, y^{*}\right) \in S^{t}$ (Mizobuchi, 2017).

Las funciones de distancia proveen la representación funcional de la tecnología subyacente al estimar la distancia radial que existe entre una combinación de insumos y productos $(x, y)$ en el periodo $t$, y la frontera de producción con la tecnología del periodo $t$, que es el límite del conjunto de tecnología $S^{t}$ (Mizobuchi, 2017). 
La función de distancia de productos del periodo $t D^{t}: \mathbb{R}_{++}^{M+N} \rightarrow \mathbb{R}_{+} \cup\{+\infty\}$ caracteriza la tecnología del periodo $t$. Está definida como sigue:

$$
D^{t}\left(x_{t}, y_{t}\right) \equiv \inf \left\{\theta>0:(x, y / \theta) \in S^{t}\right\}
$$

Por definición, la función de distancia de insumos es homogénea en primer grado en el insumo $x$ y la función de distancia de productos es homogénea en primer grado en producto $y$, como se indica a continuación:

$$
D^{t}\left(x_{t}, \lambda y_{t}\right)=\lambda d^{t}(x, y)
$$

Los retornos constantes a escala para para $t=0$ y 1 se encuentran definidos como:

$$
\left(x_{t}, y_{t}\right) \in S^{t} \text { implica }\left(\lambda x_{t}, \lambda y_{t}\right) \in S^{t} \text { para todo } \lambda>0
$$

En este sentido, cuando la tecnología sigue retornos constantes a escala, la función de distancia de producto es recíproca a la función de distancia de insumo y se expresa como sigue:

$$
d^{t}(x, y)=\frac{1}{D^{t}(x, y)}
$$

Bajo este contexto, se define un IM orientado al producto (índice de cantidad de producto, $I M_{O}^{t}$ ), a manera de determinar la cantidad máxima de productos que puede conseguirse dada una cantidad de insumos disponible, y donde la tecnología en $t$ se considera como referencia, como:

$$
I M_{O}^{t}=\frac{D_{O}^{t}\left(x_{t+1}, y_{t+1}\right)}{D_{O}^{t}\left(x_{t}, y_{t}\right)}
$$

De la misma manera puede definirse un IM cuya tecnología de referencia está basada en el periodo $t+1$ :

$$
I M_{O}^{t+1}=\frac{D_{O}^{t+1}\left(x_{t+1}, y_{t+1}\right)}{D_{O}^{t+1}\left(x_{t}, y_{t}\right)}
$$

En ambos casos, $I M_{O}^{t}$ e $I M_{O}^{t+1}$ calculan diferentes índices de productividad debido a que sus tecnologías de referencia pueden variar (Färe et al., 1998).

La media geométrica de los IM en (6) y (7) se emplea en la estimación de los cambios en la productividad en un IM con orientación al producto. Debido a que se trata de una razón de funciones de distancia que pueden modelizarse de manera que resulte equivalente a las medidas de eficiencia técnica de Farrell (1957), esto permite que los CPTF se descompongan y se aíslen las distintas fuentes 
que originan estos cambios (Lovell, 2003), con ello se obtiene de manera simultánea la descomposición en cambios de eficiencia (catching up) y cambios de tecnología (frontier shift) a lo largo del tiempo (Färe et al., 1994) como se indica a continuación:

$$
I M_{O}^{t, t+1}\left(x_{t}, y_{t}, x_{t+1}, y_{t+1}\right)=\left[\frac{D_{O}^{t}\left(x_{t+1}, y_{t+1}\right)}{D_{O}^{t}\left(x_{t}, y_{t}\right)} \cdot \frac{D_{O}^{t+1}\left(x_{t+1}, y_{t+1}\right)}{D_{O}^{t+1}\left(x_{t}, y_{t}\right)}\right]^{1 / 2}
$$

Donde $D_{O}^{t}\left(x_{t+1}, y_{t+1}\right)$ y $D_{O}^{t}\left(x_{t}, y_{t}\right)$ representan funciones de distancia de las observaciones de la tecnología del periodo $\mathrm{t}+1$ y del periodo $\mathrm{t}$, $\mathrm{y}$ de las observaciones referentes a la tecnología del periodo $t y$ del periodo $t+1$, respectivamente

Para interpretar los resultados de las estimaciones, en el caso de un IM orientado al producto, se observa una mejora o un incremento en la productividad del periodo $t$ al periodo $t+1$ cuando es mayor a la unidad $\left(I M_{O}^{t, t+1}>1\right)$, una pérdida en la productividad cuando es menor a la unidad $\left(I M_{O}^{t, t+1}<1\right)$ y sin cambios en la productividad cuando es igual a la unidad $\left(I M_{O}^{t, t+1}=1\right)$.

De la metodología de Färe et al. (1994) se obtiene la descomposición de los CPTF estimados a través del IM en (8) como se expresa a continuación:

$$
I M_{O}^{t, t+1}\left(x_{t}, y_{t}, x_{t+1}, y_{t+1}\right)=\frac{D_{O}^{t+1}\left(x_{t+1}, y_{t+1}\right)}{D_{O}^{t}\left(x_{t}, y_{t}\right)}\left[\frac{D_{O}^{t}\left(x_{t+1}, y_{t+1}\right)}{D_{O}^{t+1}\left(x_{t+1}, y_{t+1}\right)} \cdot \frac{D_{O}^{t}\left(x_{t}, y_{t}\right)}{D_{O}^{t+1}\left(x_{t}, y_{t}\right)}\right]^{1 / 2}
$$

El primer componente estima el cambio en eficiencia (técnica), lo que implica un acercamiento a la frontera eficiente, muestra la relación entre la eficiencia en el periodo $t+1 \mathrm{y}$ la eficiencia en el periodo $t$ :

$$
\text { eficiencia }=\frac{D_{O}^{t+1}\left(x_{t+1}, y_{t+1}\right)}{D_{O}^{t}\left(x_{t}, y_{t}\right)}
$$

El segundo componente estima el cambio en tecnología, o cambios en la frontera, como una media geométrica de las razones entre los periodos $t$ y $t+1$ :

$$
\text { tecnología }=\left[\frac{D_{O}^{t}\left(x_{t+1}, y_{t+1}\right)}{D_{O}^{t+1}\left(x_{t+1}, y_{t+1}\right)} \cdot \frac{D_{O}^{t}\left(x_{t}, y_{t}\right)}{D_{O}^{t+1}\left(x_{t}, y_{t}\right)}\right]^{1 / 2}
$$

La interpretación de cada componente sigue la misma lógica del IM, donde una mejora se denota si el resultado es mayor a la unidad, una pérdida si es menor a la unidad y sin cambios cuando es igual a la unidad.

Una de las particularidades del IM está en que permite que los cambios en eficiencia y los cambios en la tecnología puedan ir en direcciones opuestas. Es decir, un incremento de eficiencia puede llevar de la mano un deterioro de tecnología, y viceversa. Asimismo, ambos componentes pueden ir en la misma dirección. 
Bajo este escenario, y a través de su descomposición, el IM ayuda a conocer cuál es la fuente de los CPTF observados dentro de un mismo grupo de DMU entre dos períodos de tiempo distintos, basado en una técnica no paramétrica que no requiere asumir una forma funcional de la frontera de producción, tampoco precisa información sobre los precios de mercado de los insumos ni de los productos, ni supuestos restrictivos sobre minimización de costos o maximización de beneficios (Salinas et al., 2003).

Cabe destacar que en esta investigación se emplea el supuesto de retornos constantes a escala para conservar las propiedades de CPTF del IM (Färe et al., 1994), situación que se pierde en presencia de retornos variables a escala (Kerstens \& Van De Woestyne, 2014).

\subsection{Datos y variables}

Al emplear las técnicas no paramétricas en las estimaciones de la PTF, la selección de variables se convierte en una de las fases más importantes, ya que los resultados que se obtengan dependerán en cierta medida de la elección de los insumos y los productos. Aunado a lo anterior, no se cuenta con alguna prueba de tipo estadístico que consiga disentir sobre el ajuste de las variables a incluir en las estimaciones. A pesar de ello, es necesario hacer una selección de variables de acuerdo con la mejor aproximación posible de la realidad que se intenta explicar, de manera que deberán seguirse los parámetros conceptuales y empíricos que muestren evidencia de la relación que guardan insumos y productos (Dyson et al., 2001).

Dentro del análisis empírico de los CPTF se utiliza un panel correspondiente a los veintinueve subsectores que conforman el sector manufactura en Colombia, con información de 2005 a 2016 tomada, principalmente, de las encuestas industriales que realiza el Departamento Administrativo Nacional de Estadística (DANE) en Colombia. La Encuesta Anual Manufacturera (EAM) es el principal insumo de este trabajo, la cual contiene información sobre la actividad económica de todos los sectores industriales que conforman la manufactura colombiana considerados como las unidades de análisis dentro de esta investigación.

Estas encuestas se empalmaron y homogenizaron a lo largo de la serie de acuerdo con el código Clasificación Internacional Industrial Uniforme (CIIU) mediante un proceso de agregación para poder hacerlos comparables en el tiempo. Para el tramo temporal que va de 2005 a 2013, la CIIU responde a la Revisión 3 (Rev. 3) y desagrega a la manufactura colombiana en 63 grandes ramas, mientras que, para los años posteriores a 2013 y hasta 2016 se siguen los lineamientos de la Revisión 4 (Rev. 4) que clasifica a la manufactura en 56 ramas. La Rev. 3 y la Rev. 4 se empalman y homogenizan al nivel de 29 grandes ramas industriales (DMU), con ello se evitan confusiones en el análisis y permite reunir en 29 divisiones claras y no repetitivas a las ramas industriales (ver Anexo 1).

A partir de 2017 el CIIU introduce la Revisión 4 A.C., aplicable para información de 2017 y 2018, con la cual se crean nuevas divisiones para la industria manufacturera, por lo que se lleva a nivel de división (dos dígitos) de componentes clasificados antes a nivel de grupo (tres dígitos), lo que va más allá de una mera reclasificación de los subsectores, con lo cual los últimos dos años mencionados con antelación se excluyen de la muestra con la finalidad de evitar serios problemas de 
agregación, quedando así un panel de 29 subsectores manufactureros con información de 2005 a 2016.

A continuación, se describe brevemente las industrias que sufrieron modificaciones que habrán de considerarse al interpretar los niveles de productividad.

- Los códigos 353 y 359 de la Rev. 3 son sumados ya que corresponden a dos ramas que hacen parte del mismo sector de "Fabricación de Equipos de Transporte" y que la Rev. 4 las contenía en el código 300.

- Después de 2013 el código 369 de la Rev. 3 es reclasificado en el código 390 de la Rev. 4 "Otras industrias" por ser esta una industria poco representativa en el total de la manufactura. De manera que, para la serie 2005-2016 se han sumado los códigos 385+390 entendidos como una misma industria.

- El código 160 de la Rev. 3, productos de Tabaco, después de 2013 también fue reclasificado en el código 390 "otras industrias" dado el cierre de la principal tabacalera del país, una vez evidenciadas pérdidas en los periodos anteriores a 2013.

- El código 361 y 369 de la Rev. 3, Muebles y Otras industrias, después de 2013 también fueron reclasificados en el código 390 "otras industrias" dado el cierre de la principal tabacalera del país, una vez evidenciadas pérdidas en los periodos anteriores a 2013.

- $\quad \mathrm{Al}$ sector de "Procesamiento y conservación de frutas, legumbres, hortalizas" se añade el de "Procesamiento de Aceites vegetales".

- Al sector de "Molinería y almidones y sus productos" se añade el sector de "Panadería y refinados".

- El sector de "Textiles" ahora incluye las industrias que la Rev.3 desagregaba por tipo de Tela (hilos de seda, hilos de fibras sintéticas, tejidos textiles). Igual sucede con el sector de "Confecciones", el cual tiene en cuenta todos los tipos de Confecciones que se realicen.

- El sector de "Sustancias químicas" después de 2013 con la Rev.4 se incluía el sector de farmacéuticos, de manera que antes de 2013 se suman los códigos 241+243.

- En el sector de maquinaria y equipo, se incluye tanto a la maquinaria general, como a la especializada (por ejemplo, calderas).

Finalmente, de los 29 subsectores antes descritos se analizarán variables relacionadas con la capacidad de la industria para generar riqueza, así como el nivel de insumos utilizados para producirla, los cuales constituye la principal fuente de Productividad. A continuación, en la Tabla 1 se relacionan las principales variables a utilizar y una breve descripción.

Tabla 1. Definición de variables

\begin{tabular}{|c|c|c|c|}
\hline \multicolumn{1}{|c|}{ Etiqueta } & \multicolumn{2}{|c|}{ Variable } & Descripción \\
\hline \multirow{3}{*}{ Producto } & $Y_{1}$ & Valor agregado & $\begin{array}{c}\text { Valor real generado por una } \\
\text { industria una vez descontada } \\
\text { a la producción el consumo } \\
\text { de materias primas y otros } \\
\text { insumos en el año t. }\end{array}$ \\
\hline
\end{tabular}




\begin{tabular}{|c|c|c|c|}
\hline Insumos & $X_{1}$ & Sueldos y Salarios & $\begin{array}{c}\text { Salario real total pagado a } \\
\text { los trabajadores contratados } \\
\text { en el año t. }\end{array}$ \\
\cline { 2 - 4 } & $X_{2}$ & $\begin{array}{c}\text { Consumo } \\
\text { Intermedio }\end{array}$ & $\begin{array}{c}\text { Valor real de materias } \\
\text { primas, energía y otros } \\
\text { insumos empleados en la } \\
\text { producción del año t. }\end{array}$ \\
\hline$X_{3}$ & Stock de Capital & $\begin{array}{c}\text { Inversión neta en } \\
\text { Maquinaria y equipo } \\
\text { realizada por la industria en } \\
\text { el año t + el acervo de capital } \\
\text { en el año t-1 }\end{array}$ \\
\hline
\end{tabular}

Fuente: Elaboración propia de los autores con información del DANE,

Encuesta Anual Manufacturera (EAM) y Encuesta de Desarrollo e Innovación Tecnológica (EDIT)

En el Anexo 2 se muestra la estadística descriptiva, por CIIU y con cifras expresadas en millones de pesos colombianos, deflactadas con el Índice de Precios al Consumidor con año base 2012, del producto y los insumos empleados para la estimación del IM en la manufactura colombiana de 2005 a 2016.

\section{Resultados y Discusión}

En esta investigación se analiza el desempeño de veintinueve subsectores productivos pertenecientes al sector manufacturero colombiano a lo largo de doce años, de 2005 a 2016, a través de la estimación del IM con orientación al producto y asumiendo retornos constantes a escala. Para la interpretación de los CPTF se emplea el siguiente criterio, un $I M>1$ corresponde a un incremento (mejora) en la productividad de un periodo a otro, mientras que un $I M<1$ indica una caída (deterioro) en la productividad, finalmente un $I M=1$ muestra que no hay cambio alguno en la productividad. Lo mismo aplica con los componentes de tecnología y eficiencia, respectivamente.

Los cambios en eficiencia son interpretados como un desplazamiento de las DMU hacia la frontera eficiente de producción a lo largo del tiempo, mientras que los cambios en la tecnología conllevan un desplazamiento de la frontera originado por el progreso tecnológico de las DMU. En este sentido, una mejora en el componente de eficiencia indica mejores prácticas en el proceso de producción, y una mejora en el componente tecnológico implica innovaciones que desplazan a la frontera de producción.

El análisis de los resultados que aquí se presenta es acompañado de algunas reflexiones sobre las condiciones del escenario económico que aconteció al sector manufacturero colombiano a lo largo del periodo de estudio. El IM a través de su descomposición permite identificar la fuente de los cambios en la productividad con el paso del tiempo y atribuir dicho cambio al componente de eficiencia o al componente de tecnología, sin embargo, el IM de manera aislada no permite medir el impacto de otras variables explicativas en la productividad. 


\subsection{Productividad de la manufactura colombiana}

La Figura 1 muestra el resumen de las medias anuales del IM. Los resultados de las estimaciones para todo el periodo (2005-2016) muestran un cambio negativo promedio en la PTF para todo el sector manufacturero de $1.1 \%$, derivado de un retroceso en la tecnología del $2.2 \%$, mientras que el componente de eficiencia contrarrestó esta pérdida con un incremento de 1.1\%.

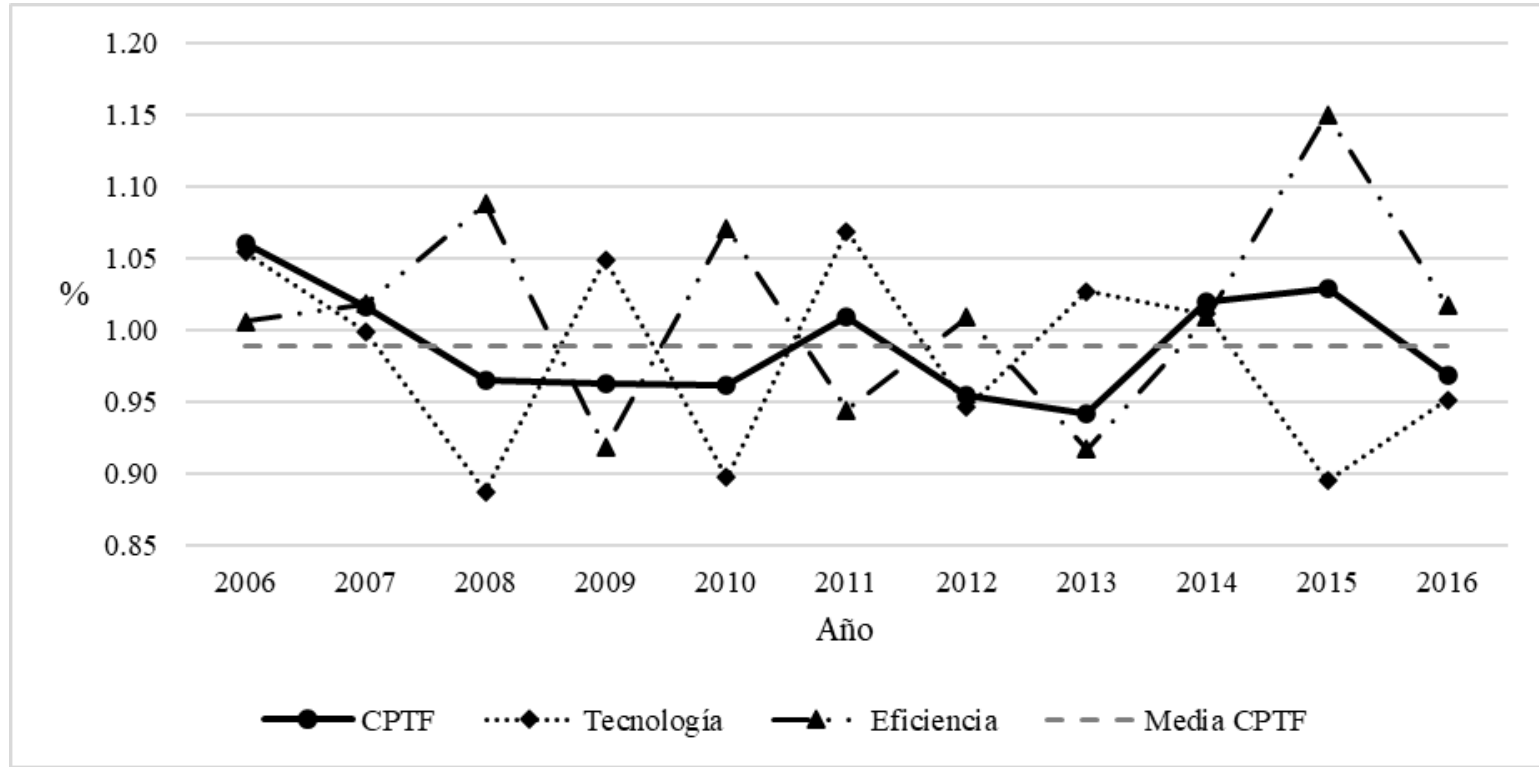

Figura 1. Medias anuales de la evolución del índice de Malmquist: 2005-2016 Fuente: Elaboración propia de los autores con base en las estimaciones.

Durante los años previos a la recesión económica de 2008 se observan pequeñas mejoras en la productividad promedio del sector manufacturero. En 2006 se alcanzó el mejor desempeño de todo el periodo de estudio con una ganancia media de 6\% (ver Figura 1), originada por una mejora del $5.4 \%$ en el componente tecnológico y del $0.6 \%$ en el componente de eficiencia. Por otra parte, en 2007 la mejora de 1.6\% promedio de la PTF se debió a una ganancia de eficiencia en 1.8\%, contrarrestada por un decremento en el componente tecnológico.

A partir de la crisis económica mundial los niveles de productividad del sector presentaron resultados mixtos (ver Figura 1), siendo el año 2013 el periodo de peor desempeño con una caída del 5.8\%, debida a una pérdida de eficiencia del 8.3\%. El año 2012 tampoco fue la excepción y precedió al 2013 con un decremento medio en las variaciones de los CPTF de 4.5\%, originado por el deterioro del componente tecnológico en 5.4\%.

Se observa, en la Figura 1, que el movimiento cíclico y finalmente negativo (0.9899) que mostró la productividad media manufacturera durante 2005-2016 aunque se debió a diversas razones intrínsecas a la eficiencia y el cambio tecnológico, y que aquí no permiten ser plenamente identificables, cabe remarcar que, aparentemente, el uso generalizado por la industria de los factores productivos (capital, trabajo, e insumos intermedios) de manera eficiente fue la razón que más repercutió en estabilizar el indicador a lo largo de los años y reducir el impacto negativo que 
ejercieron los escasos cambios tecnológicos introducidos por la manufactura colombiana durante el periodo de estudio.

Otro hallazgo identificado se refiere al momento en el que la eficiencia y el cambio tecnológico destacan en la explicación de la PTF manufacturera. Por ejemplo, en la Figura 1 se observa que los años en el que el factor de eficiencia aumentó (fue mayor a 1) le presidió un periodo en el que esta misma cayó y se incrementó el cambio tecnológico. Desde 2006, periodo de mayor PTF, y a excepción de 2014, los componentes de eficiencia y de cambio tecnológico nunca fueron de la mano o se conjugaron para que la manufactura ganara productividad, por el contrario, se observa que en la manufactura coexistió una estrategia basada en periodos con un alto cambio tecnológico, posiblemente en forma adquisición de maquinaria y equipo, para que en periodos posteriores fueran utilizados para hacer más eficientes los demás factores de producción, trabajo y bienes intermedios.

De manera que, lo anterior indica que el cambio tecnológico o la introducción de innovaciones en la manufactura colombiana no podrían concebirse, aún, como factores críticos de éxito para la productividad y el crecimiento económico del sector y que dicho factor tecnológico sigue estando en fase de maduración, aunque con serias dificultades después de la crisis hipotecaria, dada la natural dependencia del sector por los tradicionales factores productivos de trabajo, capital fijo y bienes intermedios.

Dado que, a 2017, tan solo el $20 \%$ de las firmas que constituyen la industria manufacturera cuentan con cierta tecnificación de sus procesos y carecen de una efectiva adaptación de las Tecnologías de la Información y la Comunicación, TICs, (DANE, 2018), es imperante que hoy los hacedores de política se centren en promover el uso de estas tecnologías TIC's dada, en primera instancia, su influencia insoslayable en la comunicación en pleno siglo XXI y, en segunda instancia, dadas las necesidades de interconectividad que implica hoy el comercio mundial de la producción, donde el sector manufacturero colombiano puede aprovechar la oportunidad de integrarse a los eslabones más avanzados de la cadenas de valor del mundo en los sectores hasta ahora desarrollados o con potencial de desarrollo.

\subsection{Productividad de los subsectores de la manufactura colombiana}

El 73\% de la manufactura (ver Tabla 2) demostró que, en periodos de crisis, si bien el cambio tecnológico era riesgoso e incluso poco pertinente, la eficiencia de los recursos permitió sobrellevar y, más tarde, rebasar los periodos críticos de 2007 y 2012 que vivió la manufactura colombiana. Por su parte, el cambio tecnológico, como era natural, solo afectó de manera positiva, aunque intermitente, a la productividad en los periodos de postcrisis.

Por otro lado, del análisis por subsector (ver Tabla 2), se destaca que el mejor desempeño durante todo el periodo de estudio se presentó en el subsector Elaboración de azúcar y panela (CIIU 157), con un avance promedio de $4.4 \%$ debido a la ganancia del componente de eficiencia de 5.2\%. Le siguen en variación promedio los subsectores de Madera y fabricación de productos de madera y de corcho, excepto muebles (CIIU 2019) con 2.9\%, Caucho (CIIU 251) con 2.7\%, Fabricación de maquinaria y equipo (CIIU 3113) con 2.7\%, y Elaboración de productos de café (CIIU 156) con 2.3\%. 
En todos los subsectores que manifestaron CPTF positivos se observó una mejora del componente de eficiencia.

El destacado crecimiento del subsector de Azúcar (CIIU 157) coincide con una alta demanda de azúcar y panela en Colombia y el mundo a lo largo del periodo analizado, así como a los precios internos regidos por el Fondo de Estabilización de Precios que pudo permitir a los pequeños y grandes productores de azúcar, caña y panela equilibrar sus ingresos ante los deprimidos precios internacionales (Asocaña, 2017). Sin embargo, se observa que la apertura de nuevos mercados en el exterior como el australiano, canadiense y el de los países de la Asociación Europea de Libre Comercio (EFTA, por sus siglas en inglés: European Free Trade Association) contribuyeron al crecimiento de las exportaciones, especialmente de panela, situación que pudiera estar relacionado con mejoras en los procesos de producción de este subsector (Asocaña, 2017).

El desempeño del subsector de Madera (CIIU 2019) podría ser una respuesta a las estrategias de política pública centradas en la formación técnica de la mano de obra, a través del Servicio Nacional de Aprendizaje (SENA), para asegurar una transformación de la madera que respondiera a los nuevos conceptos de negocio de las industrias del diseño de interiores y de la construcción, quienes se basan en conceptos de "ármelo usted mismo" y los muebles tipo RTA. Asimismo, los avances alcanzados en tema de reforestación y sostenibilidad del negocio por parte de empresas y gobierno a través de la Federación Nacional de Industriales de la Madera (FEDEMADERAS), han sido clave en la integración de gran parte de la cadena de valor de la madera y dando responsabilidades para que operen de manera coordinada (Rueda et al., 2016) y en pro de reducir las importaciones de su insumo principal, la madera.

Similar a lo anterior, el crecimiento y el desempeño del sector de Caucho (CIIU 251) y de Vidrio (CIIU 261) están vinculados al dinamismo y al buen comportamiento de sectores como el de la construcción y de otros sectores de servicios. También se hallaron como sectores productivos el de Químicos (CIIU 2413), Papel y cartón (CIIU 210) y, en menor medida, Maquinaria y equipo (CIIU 3113) los cuales requieren a los primeros como materia prima de sus productos finales (Mendoza \& Oliveros, 2018).

Tabla 2. Medias anuales por CIIU de la evolución del índice de Malmquist: 2005-2016

\begin{tabular}{|c|c|c|c|c|}
\hline DMU & CIIU & CPTF & Tecnología & Eficiencia \\
\hline $\mathbf{6}$ & $\mathbf{1 5 7}$ & 1.044 & 0.992 & 1.052 \\
\hline $\mathbf{2 2}$ & $\mathbf{2 0 1 9}$ & 1.029 & 0.989 & 1.040 \\
\hline $\mathbf{1 2}$ & $\mathbf{2 5 1}$ & 1.027 & 0.995 & 1.033 \\
\hline $\mathbf{2 7}$ & $\mathbf{3 1 1 3}$ & 1.027 & 0.989 & 1.039 \\
\hline $\mathbf{5}$ & $\mathbf{1 5 6}$ & 1.023 & 0.970 & 1.055 \\
\hline $\mathbf{1 0}$ & $\mathbf{2 1 0}$ & 1.019 & 0.985 & 1.034 \\
\hline $\mathbf{1 9}$ & $\mathbf{1 7 1 4}$ & 1.019 & 1.000 & 1.019 \\
\hline $\mathbf{1 5}$ & $\mathbf{2 6 9}$ & 1.018 & 0.987 & 1.032 \\
\hline $\mathbf{1 4}$ & $\mathbf{2 6 1}$ & 1.017 & 0.995 & 1.023 \\
\hline $\mathbf{3}$ & $\mathbf{1 5 3}$ & 1.015 & 0.970 & 1.047 \\
\hline $\mathbf{2 0}$ & $\mathbf{1 7 5 8}$ & 1.014 & 1.006 & 1.007 \\
\hline $\mathbf{1}$ & $\mathbf{1 5 1}$ & 1.010 & 0.960 & 1.052 \\
\hline
\end{tabular}




\begin{tabular}{|c|c|c|c|c|}
$\mathbf{2}$ & $\mathbf{1 5 2}$ & 1.005 & 0.970 & 1.036 \\
\hline $\mathbf{2 4}$ & $\mathbf{2 4 1 3}$ & 1.002 & 0.974 & 1.029 \\
\hline $\mathbf{4}$ & $\mathbf{1 5 5}$ & 0.999 & 0.954 & 1.047 \\
\hline $\mathbf{1 8}$ & $\mathbf{3 6 9}$ & 0.999 & 0.985 & 1.014 \\
\hline $\mathbf{7}$ & $\mathbf{1 5 8}$ & 0.989 & 0.981 & 1.008 \\
\hline $\mathbf{1 3}$ & $\mathbf{2 5 2}$ & 0.989 & 0.988 & 1.000 \\
\hline $\mathbf{8}$ & $\mathbf{1 5 9}$ & 0.985 & 0.985 & 1.000 \\
\hline $\mathbf{2 5}$ & $\mathbf{2 7 2 8}$ & 0.985 & 0.973 & 1.012 \\
\hline $\mathbf{2 8}$ & $\mathbf{3 4 1 3}$ & 0.983 & 0.953 & 1.032 \\
\hline $\mathbf{2 6}$ & $\mathbf{2 9 1 3}$ & 0.978 & 0.995 & 0.983 \\
\hline $\mathbf{9}$ & $\mathbf{1 9 2}$ & 0.966 & 1.004 & 0.962 \\
\hline $\mathbf{1 7}$ & $\mathbf{3 6 1}$ & 0.961 & 0.992 & 0.969 \\
\hline $\mathbf{2 3}$ & $\mathbf{2 2 1 3}$ & 0.960 & 0.996 & 0.963 \\
\hline $\mathbf{2 1}$ & $\mathbf{1 9 1 3}$ & 0.946 & 0.989 & 0.956 \\
\hline $\mathbf{1 6}$ & $\mathbf{2 7 1}$ & 0.928 & 0.980 & 0.947 \\
\hline $\mathbf{2 9}$ & $\mathbf{3 5 1 9}$ & 0.892 & 0.943 & 0.946 \\
\hline $\mathbf{1 1}$ & $\mathbf{2 3 2}$ & 0.880 & 0.880 & 1.000 \\
\hline & Media & $\mathbf{0 . 9 8 9}$ & $\mathbf{0 . 9 7 8}$ & $\mathbf{1 . 0 1 1}$ \\
\hline
\end{tabular}

Fuente: Elaboración propia de los autores con base en las estimaciones.

Nota: Todas las medias del IM son medias geométricas.

A tales relaciones de vinculación intersectorial también responden los sectores de Textiles (CIIU 1714) y Confecciones (CIIU 1758) cuyas productividades vieron crecimiento positivos (ver Tabla 3), aquí se ha observado una mejora en la calidad y los precios de los insumos, especialmente de la mano de obra formal e informal, y que, a diferencia de los sectores antes mencionados, estos sectores se han visto fortalecidos por sus continuas inversiones en reconversión del capital fijo, capacitación del capital humano y el apoyo, indudable, de entidades como ProColombia en posicionar los textiles y las confecciones colombianas en el mundo (SURA, 2013).

En contraste, el peor desempeño de la PTF a lo largo de los once años fue observado en el subsector Coquización, fabricación de productos de la refinación del petróleo y actividad de mezcla de combustibles (CIIU 232), con un retroceso promedio de 12\% como consecuencia de una caída en el componente tecnológico y la invariabilidad de la eficiencia. La caída del sector coincide con un desplome en la rentabilidad del sector originado por el declive en la demanda internacional de hidrocarburos y la baja incesante de los precios del petróleo, hecho que afectó a la economía y la industria colombiana en su conjunto, dado que el petróleo explica el $9 \%$ del PIB y cerca del 70\% de las exportaciones del país.

La caída de la rentabilidad ya mencionada también se observó en el subsector de Bebidas (CIIU 159), donde la reforma tributaria de 2016 y la Ley de licores, además de aumentar el IVA de las bebidas alcohólicas del 16\% al 18\%, gravó las utilidades y la rentabilidad, ya decadentes, del sector ante la disminución, más que proporcional, en la demanda por el incremento de los precios (Lancheros \& Ruiz, 2015). Asimismo, las bebidas alcohólicas, que representan el 60\% del subsector 
de Bebidas, se vieron afectadas por la caída en la demanda local y regional ocasionada por la reducción en el consumo de bebidas azucaradas a consecuencia del debate político y social vivido en Colombia (2016-2018) con respecto a gravar las bebidas azucaradas para mitigar los efectos adversos que tienen en la salud.

Otros subsectores que presentaron un nivel bajo de productividad y, al mismo tiempo, un negativo cambio en el componente tecnológico fueron Otro equipo de Transporte (CIIU 3519), cuya caída de este último fue ligeramente superior a la del componente de eficiencia, Productos de panadería y molinería (CIIU 155), Joyas, instrumentos deportivos, musicales (CIIU 369), Otros alimentos (CIIU 158), Plástico (CIIU 252) y Bebidas (CIIU 159), donde los dos últimos sufrieron caídas del $1.2 \%$ y $1.5 \%$ en la tecnología, respectivamente, e invariabilidad de la eficiencia.

A lo anterior, también se podrían sumar factores de incertidumbre e inestabilidad económica provocados por los efectos climáticos de fenómeno del Niño y que afectaron en gran medida al subsector de Productos alimenticios (CIIU 158), el sector de Molienda, almidones y panadería (CIIU 155) en especial a la producción de arroz, y una vez más, al sector de Bebidas (CIIU 159).

Asimismo, la caída de la PTF en los subsectores de Hierro y acero (CIIU 271), Curtido, recurtido de cuero y sus productos (CIIU 1913), Edición e impresión (CIIU 2213), Muebles (CIIU 361), Calzado (CIIU 192) y Aparatos y equipos eléctrico (CIIU 2913) se debió a una caída superior en la eficiencia con respecto de lo ocurrido con el componente tecnológico, llamando la atención sobre el papel de los insumos para este grupo de subsectores en Colombia.

Finalmente, la apreciación del tipo de cambio y que disminuyó las exportaciones a razón de la competencia internacional, especialmente China, y la dependencia de país por hidrocarburos, ha llevado a elevar el costo de los productos colombianos en el exterior, como principal síntoma de la enfermedad holandesa y la desindustrialización temprana, y provocado una reducción de las inversiones en materia de capital, trabajo y materias primas en los sectores manufactureros (F. Álvarez et al., 2018). Lo anterior aunado a la inestabilidad de la economía colombiana ante los anuncios constantes de reformas tributarias y financieras, traen consigo una desestabilización de la confianza de los industriales en el futuro económico del país, ello pudiera estar vinculado con alrededor del 70\% de los subsectores menos productivos del país, como Equipo de transporte (CIIU 3519), Edición e Impresión (CIIU 2213), Plásticos (CIIU 252), Calzado (CIIU 192), Cueros (CIIU 1913), Metales preciosos (CIIU 2728), entre otros.

\section{Conclusiones}

El sector manufacturero colombiano ha buscado generar valor agregado al enfocarse en el desarrollo de capacidades tecnológicas, como la compra de maquinaria especializada, además de centrarse en la productividad por medio de la reducción de costos, así como en la eficiencia de procesos de comercialización y exportación. Sin embargo, esta industria ha manifestado dificultades para recuperarse tras la crisis de 2008, sin alcanzar tasas de crecimiento similares de años previos.

Dada la relevancia histórica de la industria manufacturera colombiana en la producción real de las diferentes ramas económicas, que llegó a representar alrededor del 35\% del total, en este trabajo se analizan empíricamente los cambios en la PTF a través de la estimación del IM con la finalidad de comprender la dinámica del sector manufacturero. Entre las ventajas que brinda esta metodología, se encuentra la descomposición de la PTF en cambios de eficiencia (catching up) y 
cambios de tecnología (frontier shift) a lo largo del tiempo, y que estos cambios pueden ir, o no, en la misma dirección.

Para dar seguimiento a la evolución de la productividad en la manufactura, lo ideal es contar con información desagregada a nivel empresa y abarcar el mayor número de años para un mejor análisis. Derivado de la falta de información al nivel mencionado con antelación, se consideró como unidad de análisis a los subsectores (ramas industriales) que integran la manufactura durante 20052016.

Los resultados de la investigación muestran que, en promedio y a lo largo del período de estudio, el sector manufacturero presentó cambios negativos en la productividad (1.1\%), siendo el componente tecnológico el origen de estos, por tanto, se confirma una desindustrialización temprana de la manufactura colombiana, donde sólo el 48\% del sector consiguió cambios positivos en la PTF. A partir de 2006, año con mayor PTF y excepto en 2014, los componentes de eficiencia y de tecnología no fueron de la mano, se observa entonces que algunos años manifestaron progreso tecnológico y que posteriormente presentaron ganancias de eficiencia. Este intercambio, evidentemente, es muestra de la intermitencia misma de las condiciones de juego para el sector y de la falta de cohesión entre los sectores manufactureros. Como se indicó en el aparatado 3, las estrategias institucionales para promover la productividad manufacturera se centraron en el apoyo a subsectores con crecimiento sostenido, desintegrando, así, cadenas productivas y rezagando sectores aislados que bien podrían aportar a un mayor crecimiento de todos los sectores en conjunto. Esta falta de cohesión, tal como mencionan Pimentel (2018) y Basole y Narayan (2020) abre la puerta a la creación de un mercado laboral informal que le resta productividad al sector dada su tendencia al uso de mano de obra poco calificada y refuerza la desindustrialización. Así, la imposibilidad de retener mano de obra en la manufactura hace posible su contratación en sectores como el de servicios o el primario, limitando la industrialización de la economía y el desarrollo de un sector de avanzada que mejore las capacidades mismas de la mano de obra, brinde más independencia económica al país, genere más empleo y no la convierta en un importador neto de bienes que, claramente, hoy produce.

Del análisis sectorial, sobresale el subsector de Elaboración de azúcar (CIIU 157) con el mejor desempeño promedio (4.4\%) originado por una ganancia de eficiencia. Por el contrario, el subsector de Coquización, fabricación de productos de la refinación del petróleo y actividad de mezcla de combustibles (CIIU 162) obtuvo el peor desempeño promedio (12\%), derivado de un deterioro en el componente tecnológico.

Ante las relaciones de dependencia intersectorial comentadas anteriormente, en los subsectores como Caucho (CIIU 163), Vidrio (CIIU 165), Químicos (CIIU175), Papel y cartón (CIIU 161), Maquinaria y equipo (CIIU 178), Textiles (CIIU 170) y Confecciones (CIIU 171), es evidente la necesidad del trabajo mancomunado dentro del sector manufacturero que se requiere para trasladar productividades y factores productivos de unos subsectores a otros y así, se profundice en la idea de construir un tejido productivo para el país. 


\section{References}

[1] Acevedo, M., \& Ramírez, J. (2005). Diferencias Regionales En La Eficiencia Técnica Del Sector Confecciones En Colombia: Un Análisis De Fronteras Estocásticas. INNOVAR. Revista de Ciencias Administrativas $\quad y \quad$ Sociales, 15(26), 90-105. Recuperado de: https://revistas.unal.edu.co/index.php/innovar/article/view/41

[2] Álvarez, A. (2001). Concepto y medición de la eficiencia productiva. En E. Pirámide (Ed.), La medición de la eficiencia y la productividad (pp. 19-40).

[3] Álvarez, F., Eslava, M., Sanguinetti, P., Toledo, M., Alves, G., Daude, C., \& Allub, L. (2018). RED 2018. Instituciones para la productividad: hacia un mejor entorno empresarial. (Corporación Andina de Fomento (ed.); Vol. 1). Recuperado de: http://scioteca.caf.com/handle/123456789/1343

[4] Andor, M., \& Hesse, F. (2014). The StoNED age: The departure into a new era of efficiency analysis? A monte carlo comparison of StoNED and the "oldies" (SFA and DEA). Journal of Productivity Analysis, 41(1), 85-109. https://doi.org/10.1007/s11123-013-0354-y

[5] Aparicio, J., Lovell, C. A. K., \& Pastor, J. T. (2016). Advances in Efficiency and Productivity (Vol. 249). Springer International Publishing. https://doi.org/10.1007/978-3-319-48461-7

[6] Asocaña. (2017). Informe Anual 2016-2017. Recuperado de: https://www.asocana.org/modules/documentos/14140.aspx

[7] Barrientos, J., Tobón, D., \& Gutiérrez, A. (2009). Producción y eficiencia estocástica: una aplicación a la industria del calzado en Colombia. Lecturas de Economía, 70, 165-190. https://doi.org/10.17533/udea.le.n70a2259

[8] Basole, A., \& Narayan, A. (2020). Long-run performance of the organised manufacturing sector in India: An Analysis of Sub-periods and Industry-level Trends. Economic and Political Weekly, 55(10), 35-44. Recuperado de: https://www.epw.in/journal/2020/10/special-articles/long-run-performanceorganised-manufacturing.html

[9] Boyd, G. A., \& Lee, J. M. (2019). Measuring plant level energy efficiency and technical change in the U.S. metal-based durable manufacturing sector using stochastic frontier analysis. Energy Economics, 81(March), 159-174. https://doi.org/10.1016/j.eneco.2019.03.021

[10] Caves, D. W., Christensen, L. R., \& Diewert, W. E. (1982). The Economic Theory of Index Numbers and the Measurement of Input, Output, and Productivity. Econometrica, 50(6), 1393-1414. https://doi.org/10.2307/1913388

[11] Clavijo, S., Vera, A., \& Fandiño, A. (2012). La desindustrialización en Colombia: Análisis cuantitativo de sus determinantes (Asociación Nacional de Instituciones Financieras (ed.)). Impresione C. Recuperado de: https://www.anif.com.co/sites/default/files/uploads/Anif-Desindustrializacion-12.pdf

[12] Coelli, T. J., Prasada Rao, D. S., O’Donnell, C. J., \& Battese, G. E. (2005). An introduction to efficiency and productivity analysis. En An Introduction to Efficiency and Productivity Analysis. Springer US. https://doi.org/10.1007/b136381

[13] CPC. (2016). Productividad, la clave para el crecimiento colombiano. Recuperado de: https://compite.com.co/productividad-la-clave-del-crecimiento-para-colombia/

[14] DANE. (2017). Encuesta Anual Manufacturera $2016 . \quad$ Recuperado de: http://www.dane.gov.co/files/investigaciones/boletines/eam/boletin_eam_2016.pdf

[15] DANE. (2018). Boletín técnico Tecnologías de la Información y Comunicación en empresas 2017. Recuperado de: https://www.dane.gov.co/files/investigaciones/boletines/tic/bol_empresas_2017.pdf

[16] Day, J. Der, \& Farid, M. (2019). Assessing performance of automobile industry using malmquist productivity index with R. South African Journal of Industrial Engineering, 30(2), 61-70. https://doi.org/10.7166/30-2-2014 
REMEF (The Mexican Journal of Economics and Finance)

La productividad del sector manufacturero: caso Colombia 2005-2016

[17] De Gregorio, J. (2018). Productivity in Emerging Market Economies: Slowdown or Stagnation? (Núm. 18-12; The Policy Implications of Sustained Low Productivity Growth). Recuperado de: https://www.piie.com/publications/working-papers/productivity-emerging-market-economiesslowdown-or-stagnation

[18] de Souza, J. P. A., \& Gómez-Ramírez, L. (2018). The paradox of Mexico's export boom without growth: A demand-side explanation. Structural Change and Economic Dynamics, 47, 96-113. https://doi.org/10.1016/j.strueco.2018.08.001

[19] Delgado, W. (2019). Eficiencia técnica de las empresas manufactureras en Colombia. Revista de Ciencias Sociales, 25(2), 73-82. https://doi.org/10.31876/rcs.v25i2.27337

[20] Dyson, R. G., Allen, R., Camanho, A. S., Podinovski, V. V., Sarrico, C. S., \& Shale, E. A. (2001). Pitfalls and protocols in DEA. European Journal of Operational Research, 132, 245-259. https://doi.org/10.1016/S0377-2217(00)00149-1

[21] Eberhardt, M., \& Teal, F. (2010). Productivity analysis in global manufacturing production (Discussion Paper Series). Recuperado de: https://www.economics.ox.ac.uk/materials/papers/4729/paper515.pdf

[22] Echavarría, J., Arbeláez, M., \& Rosales, M. (2006). La productividad y sus determinantes: El caso de la industria colombiana. Desarrollo y Sociedad, 57, 77-122. https://doi.org/10.13043/dys.57.3

[23] Färe, R., Grosskopf, S., Lindgren, B., \& Roos, P. (1994). Productivity Developments in Swedish Hospitals: A Malmquist Output Index Approach. En A. Charnes, W. W. Cooper, A. Y. Lewin, \& L. M. Seiford (Eds.), Data Envelopment Analysis: Theory, Methodology, and Applications (pp. 253-272). Springer Netherlands. https://doi.org/10.1007/978-94-011-0637-5_13

[24] Färe, R., Grosskopf, S., \& Roos, P. (1998). Malmquist Productivity Indexes: A Survey of Theory and Practice. En R. Färe, S. Grosskopf, \& R. Russell (Eds.), Index Numbers: Essays in Honour of Sten Malmquist (pp. 127-190). Springer Science+Business Media, LLC. https://doi.org/10.1007/978-94-011-4858$0 \_4$

[25] Farrell, M. J. (1957). The Measurement of Productive Efficiency. Journal of the Royal Statistical Society. Series A (General), 120(3), 253-290. https://doi.org/10.2307/2343100

[26] Felipe, J., Mehta, A., \& Rhee, C. (2019). Manufacturing matters -.but it's the jobs that count. Cambridge Journal of Economics, 43(1), 139-168. https://doi.org/10.1093/cje/bex086

[27] Fernandes, A. M., \& Paunov, C. (2012). Foreign direct investment in services and manufacturing productivity: Evidence for Chile. Journal of Development Economics, 97(2), 305-321. https://doi.org/10.1016/j.jdeveco.2011.02.004

[28] Gómez, L. (2015). Diferencias en la evolución de la productividad regional en la industria colombiana: Un análisis sectorial a partir de fronteras estocásticas de producción time varying: 1992-2010. Desarrollo y Sociedad, 75, 101-152. https://doi.org/10.13043/DYS.75.3

[29] Ha, D. T. T., Kiyota, K., \& Yamanouchi, K. (2016). Misallocation and productivity: The case of Vietnamese manufacturing. Asian Development Review, 33(2), 94-118. https://doi.org/10.1162/ADEV_a_00074

[30] Haltiwanger, J., \& Eslava, M. (2017). The Drivers of Life-cycle Growth of Manufacturing Plants. Meeting Papers 1540. Recuperado de: https://economicdynamics.org/meetpapers/2017/paper_1540.pdf

[31] Kalai, M., \& Helali, K. (2016). Technical Change and Total Factor Productivity Growth in the Tunisian Manufacturing Industry: A Malmquist Index Approach. African Development Review, 28(3), 344-356. https://doi.org/10.1111/1467-8268.12210

[32] Kaldor, N. (1975). Economic Growth and the Verdoorn Law -- A Comment on Mr Rowthorn's Article. The Economic Journal, 85(340), 891-896. https://doi.org/10.2307/2230633 
[33] Karadağ, M., Önder, A. Ö., \& Deliktaş, E. (2005). Growth of factor productivity in the Turkish manufacturing industry at provincial level. Regional Studies, 39(2), 213-223. https://doi.org/10.1080/003434005200060007

[34] Kerstens, K., \& Van De Woestyne, I. (2014). Comparing Malmquist and Hicks-Moorsteen productivity indices: Exploring the impact of unbalanced vs. balanced panel data. European Journal of Operational Research, 233(3), 749-758. https://doi.org/10.1016/j.ejor.2013.09.009

[35] Kim, S., \& Han, G. (2001). A decomposition of total factor productivity growth in Korean manufacturing industries: a Stochastic Frontier Approach. Journal of Productivity Analysis, 16(3), 269-281. https://doi.org/10.1023/A:1012566812232

[36] Kumar, S. (2006). A decomposition of total productivity growth: A regional analysis of Indian industrial manufacturing growth. International Journal of Productivity and Performance Management2, 55(3/4), 311-331. https://doi.org/10.1108/17410400610653255

[37] Lancheros, R., \& Ruiz, J. (2015). Reforma Tributaria a los Licores en Colombia. Análisis detallado de riesgos y oportunidades en la industria nacional de licores. [Colegio de Estudios Superiores de Administración]. Recuperado de: https://repository.cesa.edu.co/bitstream/handle/10726/1081/MFC_392.pdf?sequence=2\&isAllowe $\mathrm{d}=\mathrm{y}$

[38] Lee, J. D., Kim, T. Y., \& Heo, E. (1998). Technological progress versus efficiency gain in manufacturing sectors. Review of Development Economics, 2(3), 268-281. https://doi.org/10.1111/1467-9361.00041

[39] Leung, H. M. (1998). Productivity of Singapore's manufacturing sector: An industry level nonparametric study. Asia Pacific Journal of Management, 15(1), 19-31. https://doi.org/10.1023/A:1015408724313

[40] Lin, Z., \& He, W. (2018). Total Factor Productivity Change of traditional manufacturing industry of Beijing. 2018 5th International Conference on Industrial Economics System and Industrial Security Engineering (IEIS), 14, 1-5. https://doi.org/10.1109/IEIS.2018.8597779

[41] Lovell, C. A. K. (2003). The decomposition of Malmquist productivity indexes. Journal of Productivity Analysis, 20(3), 437-458. https://doi.org/10.1023/A:1027312102834

[42] Mahadevan, R. (2002). No A DEA approach to understanding the productivity growth of Malaysia's manufacturing industries. Asia Pacific Journal of Management, 19, 587-600. https://doi.org/10.1023/A:1020577811369

[43] Malmquist, S. (1953). Index numbers and indifference surfaces. Trabajos de Estadistica, 4(2), 209-242. https://doi.org/10.1007/BF03006863

[44] Martínez, A., \& Ocampo, J. (2011). Hacia una política industrial de nueva generación para Colombia. D'vinni. Recuperado de: https://jaocampodotnet.files.wordpress.com/2012/03/haciaunapolc3adticaindustrialdenuevagener acic3b3n.pdf

[45] Meléndez, M., Seim, K., \& Medina, P. (2003). Productivity dinamics of the colombian manufacturing sector (Núm. 2003-23; CEDE). Recuperado de: http://economia.uniandes.edu.co/publicaciones/d2003-23.pdf

[46] Mendoza, M., \& Oliveros, D. (2018). Eficiencia de las empresas agroindustriales del sector de caucgo en Colombia: un enfoque DEA. Revista ESPACIOS, 39(51), 13-29. Recuperado de: http://www.revistaespacios.com/a18v39n51/a18v39n51p13.pdf

[47] Mizobuchi, H. (2017). A superlative index number formula for the Hicks-Moorsteen productivity index. Journal of Productivity Analysis, 48(2-3), 167-178. https://doi.org/10.1007/s11123-017-0514-6

[48] Moorsteen, R. (1961). On Measuring Productive Potential and Relative Efficiency. The Quarterly Journal of Economics, 75(3), 451-467. https://doi.org/10.2307/1885133 
[49] Muendler, M.-A. (2004). Trade, technology and productivity: a study of brazilian manufactures, 19961998 (Núm. 1148; CESifo Working Paper). Recuperado de: https://www.ifo.de/DocDL/cesifo1_wp1148.pdf

[50] Murillo-Zamorano, L. (2004). Economic efficiency and frontier techniques. Journal of Economic Surveys, 18(1), 33-45. https://doi.org/10.1111/j.1467-6419.2004.00215.x

[51] OIT. (2017). Sobre Informalidad y Productividad: Breves reflexiones para el caso del Perú. Recuperado de: $\quad$ https://www.ilo.org/wcmsp5/groups/public/---americas/---ro-lima/---srolima/documents/publication/wcms_561234.pdf

[52] Önder, A. Ö., Deliktaş, E., \& Lenger, A. (2003). Efficiency in the manufacturing industry of selected provinces in Turkey: A stochastic frontier analysis. Emerging Markets Finance and Trade, 39(2), 98113. https://doi.org/10.1080/1540496x.2003.11052537

[53] Pavcnik, N. (2002). Trade liberalization, exit, and productivity improvements: Evidence from Chilean plants. Review of Economic Studies, 69(1), 245-276. https://doi.org/10.1111/1467-937X.00205

[54] Pimentel, J. (2018). Desindustrialización, desempleo e informalidad: Un Análisis Descriptivo Para Las Principales Ciudades De Colombia [Universidad de la Salle]. Recuperado de: https://ciencia.lasalle.edu.co/economia/608

[55] Pombo, C. (1999). Productividad industrial en Colombia: una aplicación de números índices. Revista de Economía del Rosario, 2(1), 107-139. Recuperado de: https://revistas.urosario.edu.co/index.php/economia/article/view/985

[56] Puyana, A., \& Romero, J. (2013). ¿Informalidad o dualismo en las manufacturas mexicanas? Perfiles latinoamericanos, 21(41), 143-177. https://doi.org/10.18504/pl2141-143-2013

[57] Rada, C. (2007). Stagnation or transformation of a dual economy through endogenous productivity growth. Cambridge Journal of Economics, 31(5), 711-740. https://doi.org/10.1093/cje/bem004

[58] Ramírez, J. (1995). Eficiencia y productividad en la industria manufacturera colombiana 1978-1991. Coyuntura Económica, XXV(1), 129-148. Recuperado de: http://hdl.handle.net/11445/2199

[59] Rodrik, D. (2013). Unconditional Convergence in Manufacturing. Quarterly Journal of Economics, 128(1), 165-204. https://doi.org/10.1093/qje/qjs047

[60] Rueda, A., Moreno, R., \& Zúñiga, J. (2016). Guía de compra y consumo responsable de madera en Colombia. Recuperado de: http://www.cam.gov.co/images/documents/phocadownload/Pacto_madera/06. GuiaCCRM_VF.pdf

[61] Salinas, J., Pedraja, F., \& Smith, P. C. (2003). Evaluating the introduction of a quasi-market in community care. Socio-Economic Planning Sciences, 37(1), 1-13. https://doi.org/10.1016/S0038-0121(02)000423

[62] Schaefer, J., \& Clermont, M. (2018). Stochastic non-smooth envelopment of data for multi-dimensional output. Journal of Productivity Analysis, 50(3), 139-154. https://doi.org/10.1007/s11123-018-05395

[63] Shi, Y., Wang, X., \& Zhu, X. (2019). Lean manufacturing and productivity changes: the moderating role of R\&D. International Journal of Productivity and Performance Management, 69(1), 169-191. https://doi.org/10.1108/IJPPM-03-2018-0117

[64] SURA. (2013). Análisis del Comportamiento y Oportunidades del sector Sistema Moda. Recuperado de: https://www.sura.com/estrategiasComerciales/documentos/pdf/informeSectorial-sistemaModa.pdf

[65] Zhang, S., Lundgren, T., \& Zhou, W. (2016). Energy efficiency in Swedish industry: A firm-level data envelopment analysis. Energy Economics, 55, 42-51. https://doi.org/10.1016/j.eneco.2015.12.023 


\section{Anexos}

Anexo 1. Código industrial de subsectores manufactureros en Colombia: Clasificación Industrial Internacional Uniforme (CIIU)

\begin{tabular}{|c|c|c|}
\hline DMU & CIIU & Descripción \\
\hline 1 & 151 & Procesamiento y conservación de carne, pescado, crustáceos y moluscos \\
\hline 2 & 152 & $\begin{array}{l}\text { Procesamiento y conservación de frutas, legumbres, hortalizas y tubérculos / } \\
\text { Procesamiento de aceites y grasas animales y vegetales }\end{array}$ \\
\hline 3 & 153 & Elaboración de productos lácteos \\
\hline 4 & 155 & $\begin{array}{l}\text { Elaboración de productos de molinería, almidones y productos derivados del } \\
\text { almidón y panadería }\end{array}$ \\
\hline 5 & 156 & Elaboración de productos de café \\
\hline 6 & 157 & Elaboración de azúcar y panela \\
\hline 7 & 158 & Elaboración de otros productos alimenticios, incluido para animales \\
\hline 8 & 159 & Elaboración de bebidas \\
\hline 9 & 192 & Calzado \\
\hline 10 & 210 & Fabricación de papel, cartón y productos de papel y cartón \\
\hline 11 & 232 & $\begin{array}{l}\text { Coquización, fabricación de productos de la refinación del petróleo y } \\
\text { actividad de mezcla de combustibles }\end{array}$ \\
\hline 12 & 251 & Caucho \\
\hline 13 & 252 & Plástico \\
\hline 14 & 261 & Fabricación de vidrio y productos de vidrio \\
\hline 15 & 269 & Fabricación de otros productos minerales no metálicos \\
\hline 16 & 271 & Industria de hierro y acero \\
\hline 17 & 361 & Muebles \\
\hline 18 & 369 & $\begin{array}{l}\text { Otras industrias manufactureras (joyas, productos deportivos, instrumentos } \\
\text { musicales) }\end{array}$ \\
\hline 19 & 1714 & Fabricación de productos textiles \\
\hline 20 & 1758 & Fabricación de prendas de vestir \\
\hline 21 & 1913 & $\begin{array}{l}\text { Curtido y recurtido de cueros, fabricación de artículos de viaje, bolsos de } \\
\text { mano y artículos similares, y fabricación de artículos de talabartería y } \\
\text { guarnicionería, adobo y teñido de pieles }\end{array}$ \\
\hline 22 & 2019 & $\begin{array}{l}\text { Madera y fabricación de productos de madera y de corcho, excepto muebles, } \\
\text { fabricación de artículos de cestería y espartería }\end{array}$ \\
\hline 23 & 2213 & Edición e impresión \\
\hline 24 & 2413 & Fabricación de sustancias, otros productos químicos y farmacéuticos \\
\hline 25 & 2728 & Fabricación de productos de metales preciosos, no ferrosos y otros \\
\hline 26 & 2913 & $\begin{array}{l}\text { Fabricación de aparatos y equipo eléctrico, informático, radio, tv, medico, } \\
\text { óptica (motores, generadores y transformadores eléctricos, pilas, etc.) }\end{array}$ \\
\hline 27 & 3113 & Fabricación de maquinaria y equipo (general y especializada) \\
\hline 28 & 3413 & Fabricación de vehículos automotores, remolques y semirremolques \\
\hline 29 & 3519 & Fabricación de otro equipo de transporte \\
\hline
\end{tabular}

Fuente: Elaboración propia de los autores con base en DANE: Encuesta Anual Manufacturera (EAM) y Encuesta de Desarrollo e Innovación Tecnológica (EDIT) 
Anexo 2. Estadística descriptiva por CIIU: subsectores manufactureros en Colombia

\begin{tabular}{|c|c|c|c|c|c|}
\hline CIIU & Estadístico & Y1 & $\mathrm{X} 1$ & $\mathrm{X} 2$ & $\mathrm{X3}$ \\
\hline \multirow{2}{*}{151} & Media & $2,329,869.29$ & $271,938.26$ & $4,909,914.95$ & $2,324,909.31$ \\
\hline & Desv. Est. & $526,540.85$ & $69,392.94$ & $996,723.18$ & $483,238.74$ \\
\hline \multirow{2}{*}{152} & Media & $1,293,584.18$ & $171,237.96$ & $2,989,609.78$ & $2,906,855.57$ \\
\hline & Desv. Est. & $163,406.21$ & $21,929.62$ & $386,638.65$ & $983,409.22$ \\
\hline \multirow{2}{*}{153} & Media & $2,441,134.75$ & $268,798.06$ & $3,813,507.77$ & $2,573,330.97$ \\
\hline & Desv. Est. & $457,269.65$ & $76,625.81$ & $382,650.25$ & $385,344.49$ \\
\hline \multirow{2}{*}{155} & Media & $2,887,687.58$ & $368,507.62$ & $7,178,729.68$ & $3,737,553.33$ \\
\hline & Desv. Est. & $1,007,608.79$ & $166,370.29$ & $2,310,646.35$ & $1,113,905.17$ \\
\hline \multirow{2}{*}{156} & Media & $840,047.82$ & $66,626.42$ & $3,285,537.72$ & $1,334,730.95$ \\
\hline & Desv. Est. & $174,879.38$ & $9,819.51$ & $567,334.98$ & $113,905.30$ \\
\hline \multirow{2}{*}{157} & Media & $1,751,665.20$ & $181,304.90$ & $2,243,420.61$ & $5,086,687.70$ \\
\hline & Desv. Est. & $427,762.52$ & $29,087.50$ & $351,399.30$ & $859,238.37$ \\
\hline \multirow{2}{*}{158} & Media & $3,783,990.76$ & $474,537.74$ & $5,483,693.66$ & $4,497,628.28$ \\
\hline & Desv. Est. & $1,684,696.21$ & $329,267.31$ & $3,723,707.04$ & $2,235,761.25$ \\
\hline \multirow{2}{*}{159} & Media & $7,541,188.34$ & $297,114.97$ & $3,550,627.14$ & $8,684,347.63$ \\
\hline & Desv. Est. & $915,287.99$ & $48,434.62$ & $460,315.62$ & $1,233,873.56$ \\
\hline \multirow{2}{*}{192} & Media & $468,829.97$ & $111,369.07$ & $542,284.38$ & $435,117.24$ \\
\hline & Desv. Est. & $71,860.04$ & $25,922.07$ & $70,297.37$ & $249,178.50$ \\
\hline \multirow{2}{*}{210} & Media & $2,575,898.13$ & $356,939.69$ & $4,291,580.41$ & $6,894,403.80$ \\
\hline & Desv. Est. & $227,018.30$ & $24,920.91$ & $263,911.23$ & $626,482.05$ \\
\hline \multirow{2}{*}{232} & Media & $10,977,906.81$ & $2,113,316.67$ & $20,685,313.96$ & $14,580,115.23$ \\
\hline & Desv. Est. & $2,495,727.31$ & $6,807,544.79$ & $12,060,287.38$ & $5,753,956.16$ \\
\hline \multirow{2}{*}{251} & Media & $319,026.13$ & $75,985.01$ & $481,170.82$ & $864,097.32$ \\
\hline & Desv. Est. & $80,628.01$ & $9,013.37$ & $133,002.73$ & $244,033.52$ \\
\hline \multirow{2}{*}{252} & Media & $3,057,828.26$ & $559,491.05$ & $4,707,732.74$ & $5,853,356.08$ \\
\hline & Desv. Est. & $258,235.81$ & $95,092.93$ & $565,667.28$ & $926,596.55$ \\
\hline \multirow{2}{*}{261} & Media & $746,048.27$ & $110,734.16$ & $737,831.17$ & $1,683,033.71$ \\
\hline & Desv. Est. & $77,484.28$ & $15,697.77$ & $109,447.17$ & $151,893.12$ \\
\hline \multirow{2}{*}{269} & Media & $5,015,834.91$ & $453,102.03$ & $3,645,479.49$ & $10,060,542.64$ \\
\hline & Desv. Est. & $857,487.83$ & $81,943.19$ & $683,527.95$ & $2,200,764.84$ \\
\hline \multirow{2}{*}{271} & Media & $3,003,600.61$ & $243,979.66$ & $3,699,962.72$ & $7,163,957.83$ \\
\hline & Desv. Est. & $1,060,432.27$ & $29,392.99$ & $308,694.65$ & $918,204.69$ \\
\hline \multirow{2}{*}{361} & Media & $940,918.56$ & $197,870.23$ & $1,164,485.69$ & $976,179.43$ \\
\hline & Desv. Est. & $159,235.30$ & $47,874.11$ & $177,471.33$ & $325,428.77$ \\
\hline \multirow{2}{*}{369} & Media & $1,254,561.60$ & $187,515.60$ & $1,069,795.87$ & $3,140,331.59$ \\
\hline & Desv. Est. & $226,396.31$ & $68,369.18$ & $321,594.82$ & $3,943,361.02$ \\
\hline \multirow{2}{*}{1714} & Media & $1,655,781.75$ & $382,423.27$ & $2,419,303.67$ & $4,062,001.24$ \\
\hline & Desv. Est. & $220,210.69$ & $54,804.93$ & $277,536.67$ & $474,074.39$ \\
\hline \multirow{2}{*}{1758} & Media & $3,213,160.28$ & $701,905.30$ & $3,907,123.29$ & $2,712,511.95$ \\
\hline & Desv. Est. & $297,227.75$ & $38,797.68$ & $233,542.52$ & $288,315.61$ \\
\hline \multirow{2}{*}{1913} & Media & $252,132.55$ & $59,910.90$ & $402,364.78$ & $304,106.70$ \\
\hline & Desv. Est. & $28,752.09$ & $5,829.30$ & $58,386.73$ & $52,881.05$ \\
\hline \multirow{2}{*}{2019} & Media & $382,928.84$ & $60,696.48$ & $463,330.09$ & $678,259.44$ \\
\hline & Desv. Est. & $59,348.48$ & $9,890.71$ & $61,964.30$ & $65,755.15$ \\
\hline
\end{tabular}




\begin{tabular}{|l|l|r|r|r|r|}
\hline \multirow{2}{*}{$2 \mathbf{2 1 3}$} & Media & $2,082,011.97$ & $419,822.65$ & $1,754,924.80$ & $2,627,805.79$ \\
& Desv. Est. & $665,922.82$ & $102,895.43$ & $220,362.29$ & $384,901.39$ \\
\hline \multirow{2}{*}{$\mathbf{2 4 1 3}$} & Media & $11,760,815.87$ & $1,384,268.49$ & $14,507,551.49$ & $14,455,288.33$ \\
& Desv. Est. & $3,624,152.75$ & $186,431.37$ & $3,537,780.61$ & $2,589,076.37$ \\
\hline \multirow{2}{*}{$\mathbf{2 7 2 8}$} & Media & $3,112,617.09$ & $528,834.36$ & $6,969,794.59$ & $4,635,155.39$ \\
& Desv. Est. & $3,443,886.74$ & $383,212.58$ & $3,835,885.38$ & $6,896,824.87$ \\
\hline \multirow{2}{*}{$\mathbf{2 9 1 3}$} & Media & $1,570,422.17$ & $347,105.67$ & $2,316,426.34$ & $1,982,194.51$ \\
& Desv. Est. & $152,633.93$ & $60,127.68$ & $528,449.82$ & $403,936.79$ \\
\hline \multirow{2}{*}{$\mathbf{3 1 1 3}$} & Media & $1,372,119.86$ & $595,031.22$ & $1,982,905.15$ & $1,699,415.65$ \\
& Desv. Est. & $115,097.82$ & $1,038,350.73$ & $540,449.05$ & $312,526.12$ \\
\hline \multirow{2}{*}{$\mathbf{3 4 1 3}$} & Media & $1,758,741.39$ & $243,032.03$ & $4,448,889.19$ & $1,484,651.20$ \\
& Desv. Est. & $247,039.36$ & $23,098.38$ & $892,666.65$ & $263,082.14$ \\
\hline \multirow{2}{*}{$\mathbf{3 5 1 9}$} & Media & $639,835.42$ & $81,175.23$ & $1,391,016.49$ & $427,019.45$ \\
& Desv. Est. & $122,347.85$ & $15,165.27$ & $241,522.15$ & $109,964.53$ \\
\hline $\mathbf{2 0 0 5 -}$ & Media & $2,725,178.91$ & $390,157.75$ & $3,967,045.12$ & $4,064,330.63$ \\
$\mathbf{2 0 1 6}$ & Desv. Est. & $657,090.40$ & $1,187,763.53$ & $1,772,542.17$ & $1,028,379.48$ \\
\hline
\end{tabular}

Fuente: Elaboración propia de los autores con información del DANE, Encuesta Anual Manufacturera (EAM) y Encuesta de Desarrollo e Innovación Tecnológica (EDIT) 